\title{
On modular happy numbers
}

\section{Raghib Abusaris $^{1}$ and Omar Bayyati ${ }^{2}$ \\ ${ }^{1}$ Department of Epidemiology and Biostatistics \\ College of Public Health and Health Informatics}

King Saud bin Abdelaziz University for Health Science

Riyadh, Saudi Arabia

e-mail: rabusaris@yahoo.com

${ }^{2}$ College of Sciences and Health Professions

King Saud bin Abdelaziz University for Health Science

Riyadh, Saudi Arabia

Received: 27 May 2017

Revised: 26 March 2018

Accepted: 20 June 2018

\begin{abstract}
In this paper, we investigate the asymptotic behavior of the sequences generated by iterating the process of summing the modular powers of the decimal digits of a number. In particular, we identify all modular happy numbers. A number is called modular happy if the sequence obtained by iterating the process of summing the modular powers of the decimal digits of the number ends with 1 .
\end{abstract}

Keywords: Happy numbers, Sequences, Recurrence relations, Difference equations, Modular arithmetic.

2010 Mathematics Subject Classification: 39A11.

\section{Motivation}

Let $\mathbb{Z}_{+}$denote the set of positive integers and let us consider the recursively defined function $\psi: \mathbb{Z}_{+} \rightarrow \mathbb{Z}_{+}$given by

$$
\psi(x)=\left\{\begin{array}{ll}
x & \text { if } \quad x \text { is single-digit } \\
\psi(\text { sum of the digits of } x) & \text { if } x \text { is multi-digit }
\end{array} .\right.
$$


Thus, for example,

$$
\psi(8)=8, \quad \psi(13)=4, \quad \psi(56)=\psi(11)=2, \text { and } \quad \psi(271)=\psi(10)=\psi(1)=1 .
$$

This function was introduced by the second author who is a pre-med student at the King Saud bin Abdulaziz University for Health Sciences. He used the symbol F. However, we recently found out that such function was investigated by Atanassov [1,2] (see also the papers cited therein) and denoted by $\psi$. As such, from here and onward, we follow Atanossov's notation. Furthermore, Bayyati explored sequences $\left\{\psi_{m}(n)=\psi\left(n^{m}\right)\right\}_{n=1}^{\infty}$ for $m=1,2,3, \ldots$ (see the EXCEL-generated Table 1 below) and formulated some observations:

- If $n$ is a multiple of 3 , then for $m \geq 2, \psi_{m}(n)=9$.

- For $m=1,2,3, \ldots, \psi_{m}(n)$ repeats after 9 . Furthermore, the repetition starts earlier whenever $m$ is a multiple of 3 .

- For $m \geq 2, \psi_{m+6}(n)=\psi_{m}(n)$ for all $n$.

\begin{tabular}{|c|c|c|c|c|c|c|c|c|c|c|c|c|c|}
\hline $\mathbf{x}$ & $\mathbf{F}$ & $F_{2}$ & $\mathbf{F}_{3}$ & $\mathbf{F}_{4}$ & $F_{5}$ & $\mathbf{F}_{6}$ & $F_{7}$ & $F_{8}$ & $\mathbf{F}_{9}$ & $F_{10}$ & $F_{11}$ & $F_{12}$ & $F_{25}$ \\
\hline 1 & 1 & 1 & 1 & 1 & 1 & 1 & 1 & 1 & 1 & 1 & 1 & 1 & 1 \\
\hline 2 & 2 & 4 & 8 & 7 & 5 & 1 & 2 & 4 & 8 & 7 & 5 & 1 & 2 \\
\hline 3 & 3 & 9 & 9 & 9 & 9 & 9 & 9 & 9 & 9 & 9 & 9 & 9 & 9 \\
\hline \begin{tabular}{|l|}
4 \\
\end{tabular} & 4 & 7 & 1 & 4 & 7 & 1 & 4 & 7 & 1 & 4 & 7 & 1 & 4 \\
\hline \begin{tabular}{|l|}
5 \\
\end{tabular} & 5 & 7 & 8 & 4 & 2 & 1 & 5 & 7 & 8 & 4 & 2 & 1 & 5 \\
\hline \begin{tabular}{|l|}
6 \\
\end{tabular} & 6 & 9 & 9 & 9 & 9 & 9 & 9 & 9 & 9 & 9 & 9 & 9 & 9 \\
\hline \begin{tabular}{|l|}
7 \\
\end{tabular} & 7 & 4 & 1 & 7 & 4 & 1 & 7 & 4 & 1 & 7 & 4 & 1 & 7 \\
\hline \begin{tabular}{|l|}
8 \\
\end{tabular} & 8 & 1 & 8 & 1 & 8 & 1 & 8 & 1 & 8 & 1 & 8 & 1 & 8 \\
\hline \begin{tabular}{|l|}
9 \\
\end{tabular} & 9 & 9 & 9 & 9 & 9 & 9 & 9 & 9 & 9 & 9 & 9 & 9 & 9 \\
\hline \begin{tabular}{|l|}
10 \\
\end{tabular} & 1 & 1 & 1 & 1 & 1 & 1 & 1 & 1 & 1 & 1 & 1 & 1 & 1 \\
\hline \begin{tabular}{|l|}
11 \\
\end{tabular} & 2 & 4 & 8 & 7 & 5 & 1 & 2 & 4 & 8 & 7 & 5 & 1 & 2 \\
\hline 12 & 3 & 9 & 9 & 9 & 9 & 9 & 9 & 9 & 9 & 9 & 9 & 9 & 9 \\
\hline
\end{tabular}

Table 1. $F_{m}(x)=\psi_{m}(x)$ for $m=1, \ldots, 25$ and $x=1, \ldots, 12$

In this paper, we go a step further and iterate the following process.

$$
f(n)=\sum_{j=0}^{k} \psi_{m}\left(d_{j}\right)=\sum_{j=0}^{k} \psi\left(d_{j}^{m}\right) \quad \text { where } \quad n=d_{k} \cdots d_{1} d_{0}, \quad m=1,2,3, \ldots
$$

We prove, in Section 3, that the sequences generated in this way converge, more precisely are eventually constant. Following the spirit of happy numbers [3, p. 374], if the limit is 1, we call the number $n$ modular happy. Moreover, preliminary results are established in Section 2, and further applications of the proof ideas will be introduced in Section 4. We conclude in Section 5 with a summary and future directions.

\section{Preliminary results}

To be on the same page, we recall the following standard definitions; see, for example, Rosen [4, p. 241] and Weisstein [5]. 
Definition 2.1. Let $q$ be a positive integer. A sequence $a_{n}$ is said to be periodic of period $q$ if

$$
a_{n+q}=a_{n} \quad \text { for } n=1,2,3, \ldots
$$

If $q$ is the smallest such integer, it is called minimal period.

Definition 2.2. Let $a$ and $b$ be integers, and let $m$ be a positive integer. Then $a \equiv b(\bmod m)$ if and only if $a \bmod m=b \bmod m$, where $a \bmod m=a-m\lfloor a / m\rfloor$ where $\lfloor\cdot\rfloor$ denotes the floor function, i.e., the greatest integer function.

For the proof of our main result in this section, we shall need the following well-known lemma; see Rosen (2012, p. 242).

Lemma 2.1. Let $m$ be a positive integer and let $a$ and $b$ be integers. Then

$$
(a+b) \bmod m=((a \bmod m)+(b \bmod m)) \bmod m
$$

and

$$
a b \bmod m=((a \bmod m)(b \bmod m)) \bmod m .
$$

Next, we state and prove the observations mentioned in Section 1. However, we first establish the following equivalency.

Lemma 2.2. For any positive integer $n$,

$$
\psi(n) \equiv n \bmod 9
$$

More precisely,

$$
\psi(n)=\left\{\begin{array}{ll}
n \bmod 9 & \text { if } n \bmod 9 \neq 0 \\
9 & \text { if } n \bmod 9=0
\end{array} .\right.
$$

Proof. If $n$ is single-digit, then the equivalency is straight forward. So, assume $n=d_{k} \cdots d_{2} d_{0}$. By the expanded notation,

$$
n=\sum_{j=0}^{k} d_{j} 10^{j}=\sum_{j=1}^{k} d_{j}\left(10^{j}-1\right)+\sum_{j=0}^{k} d_{j} .
$$

Since the first sum is a multiple of 9 ,

$$
n \bmod 9=\left(\sum_{j=0}^{k} d_{j}\right) \bmod 9 .
$$

Hence, the result follows.

Remark 2.1. The above equivalency lemma was also observed by Atanassov [2, p. 9]

Theorem 2.1. Let $m$, $n$ be positive integers and recall $\left\{\psi_{m}(n)=\psi\left(n^{m}\right)\right\}_{n=1}^{\infty}$ for $m=1,2, \ldots$ Then 
1. If $n$ is a multiple of 3 , then for $m \geq 2, \psi_{m}(n)=9$.

2. For $m=1,2,3, \ldots$, the sequence $\left\{\psi_{m}(n)\right\}_{n=1}^{\infty}$ is periodic of period 9 .

3. If $m$ is a multiple of 3 , then the minimal period is 3 .

4. For $m \geq 2, \psi_{m+6}(n)=\psi_{m}(n)$ for all $n$.

5. For $m \geq 2, \psi_{m}(n) \neq 6$ for all $n \geq 1$.

Proof. As seen below, Lemma 2.2 plays a key role in our proof.

1. Suppose $n=3 \ell$ for some positive integer $\ell$. Since $(3 \ell)^{k}$ is a multiple of 9 for $k \geq 2$,

$$
(3 \ell)^{k} \bmod 9=0 \text {, }
$$

and so $\psi_{k}(n)=9$.

2. With the understanding that, $\psi(n)=\psi_{1}(n)$, for any $k \geq 1$, by Lemma (2.1),

$$
(n+9)^{k} \bmod 9=(n+9 \bmod 9)^{k} \bmod 9=(n \bmod 9)^{k} \bmod 9 .
$$

Therefore, $\psi_{k}(n+9)=\psi_{k}(n)$ for all $n \geq 1$.

3. Assume $k$ is a multiple of 3 . Then

$$
\begin{aligned}
(n+3)^{k} \bmod 9 & =(n+3)^{3 \ell} \bmod 9=\left((n+3)^{3}\right)^{\ell} \bmod 9 \\
& =\left(n^{3}+9 n^{2}+27 n+27\right)^{\ell} \bmod 9=\left(n^{3}\right)^{\ell} \bmod 9 \\
& =n^{k} \bmod 9 .
\end{aligned}
$$

4. Notice that $\psi_{6}(n+3)=\psi_{6}(n)$ and $\left\{n^{6}\right\}_{n=1}^{\infty} \bmod 9=\{1,1,0,1,1,0,1,1,0, \ldots\}$. Consequently,

$$
n^{k+6} \bmod 9=n^{6} n^{k} \bmod 9=n^{k} \bmod 9 .
$$

5. This part follows from Part (4) and the fact that $\psi_{k}(n) \neq 6$ for $k=2,3, \ldots, 7$.

Remark 2.2. Aside from the terminology, Theorem 2.1 was also observed by Atanassov [2].

\section{Main results}

For each positive integer $m$ and in light of Equation (1.2), consider the sequence $\left\{x_{i+1}\right\}_{0}^{\infty}$ defined by

$$
\begin{aligned}
x_{0}= & d_{k} \cdots d_{1} d_{0}=\sum_{j=0}^{k} d_{j} 10^{j} \\
x_{1}= & f\left(x_{0}\right)=\sum_{j=0}^{k} \psi\left(d_{j}^{m}\right) \\
\vdots & \vdots \\
x_{i+1}= & f\left(x_{i}\right), \quad \text { for } \quad i=0,1,2, \ldots
\end{aligned}
$$


With that in mind, our first main result in this section reads as follows:

Theorem 3.1. For each $m=1,2,3, \ldots, x_{i}$ is eventually less than 10.

Proof. Observe that if $x_{0}$ is a power of 10 , then $x_{i}=1$ for all $i \geq 1$, and hence all powers of 10 are modular happy. Furthermore, if $x_{0}=9$, then $x_{i}=9$ for all $i \geq 1$, and so multiples of 9 are not modular happy.

If $m=1$, then

$$
\begin{aligned}
& f(n)=n \quad \text { if } k=0, \text { i.e., } n=d_{0} \\
& f(n)=\sum_{j=0}^{k} d_{j}<\sum_{j=0}^{k} d_{j} 10^{j}=n
\end{aligned}
$$

Hence, by the Monotonic Convergence Theorem, the result follows.

To this end, assume that $m \geq 2$ and $n=\sum_{j=0}^{k} d_{j} 10^{j}$. Then

$$
\begin{aligned}
f(n) & =\sum_{j=0}^{k} \psi\left(d_{j}^{m}\right) \leq 9(k+1) \\
& \leq n \text { if } \quad n \geq 9(k+1) .
\end{aligned}
$$

In particular, if $k=1$, then $f(n)<n$ if $n \geq 19$. But, if $n=1 d_{0}$, then, by Theorem 2.1, $f(n)<n$ if $n \geq 10$.

Moreover, if $k \geq 2$, then

$$
\begin{aligned}
f\left(d_{k} d_{k-1} \cdots d_{0}\right) & =\psi\left(d_{k}^{m}\right)+f\left(d_{k-1} \cdots d_{0}\right) \\
& \leq 9+d_{k-1} \cdots d_{0} \quad \text { assuming the inquality holds for } k-1 \\
& =9+\left(n-d_{k} 10^{k}\right)<n
\end{aligned}
$$

Therefore, by the Principle of Mathematical Induction, $f(n)<n$ for all $k \geq 1$. This completes the proof.

The next result constitutes our second main result in this section. It characterizes the asymptotic behavior of all solution of Equation (3.1).

Theorem 3.2. If $m \equiv 2,5 \bmod 6$, then every solution of Equation (3.1) is either eventually constant or eventually periodic of period 2. Otherwise, every solution of Equation (3.1) is eventually constant.

Proof. In light of Theorem 2.1, it is enough to study $m=1,2,3,4,5,6,7$.

If $m=1$, then every solution of Equation (3.1) is eventually constant. The possible constants are $1,2, \ldots, 9$. This is true because $f(n)=n$ for all $n \in[1,9] \cap \mathbb{Z}_{+}$.

If $m=2$, then every solution of Equation (3.1) is eventually constant, namely 1,9 , or eventually periodic, namely $\{4,7\}$. This is true because

$$
\begin{array}{lll}
f(1)=1, & f(2)=4, & f(3)=9, \\
f(4)=7, & f(5)=7, & f(6)=9, \\
f(7)=4, & f(8)=1, & \text { and } \quad f(9)=9 .
\end{array}
$$


If $m=3$, then every solution of Equation (3.1) is eventually constant, namely 1,8,9. This is true because

$$
\begin{aligned}
& f(1)=1, \quad f(2)=8, \quad f(3)=9, \\
& f(4)=1, \quad f(5)=8, \quad f(6)=9, \\
& f(7)=1, \quad f(8)=8, \quad \text { and } \quad f(9)=9 \text {. }
\end{aligned}
$$

If $m=4$, then every solution of Equation (3.1) is eventually constant, namely $1,4,7,9$. This holds true because

$$
\begin{array}{lll}
f(1)=1, & f(2)=7, & f(3)=9, \\
f(4)=4, & f(5)=4, & f(6)=9, \\
f(7)=7, & f(8)=1, & \text { and } \quad f(9)=9 .
\end{array}
$$

If $m=5$, then every solution of Equation (3.1) is eventually constant, namely $1,8,9$, or eventually periodic, namely $\{2,5\}$ or $\{4,7\}$. This holds true because

$$
\begin{array}{lll}
f(1)=1, & f(2)=5, & f(3)=9, \\
f(4)=7, & f(5)=2, & f(6)=9, \\
f(7)=4, & f(8)=8, & \text { and } \quad f(9)=9 .
\end{array}
$$

If $m=6$, then every solution of Equation (3.1) is eventually constant, namely 1,9. This holds true because

$$
\begin{aligned}
& f(1)=1, \quad f(2)=1, \quad f(3)=9, \\
& f(4)=1, \quad f(5)=1, \quad f(6)=9, \\
& f(7)=1, \quad f(8)=1, \quad \text { and } \quad f(9)=9 \text {. }
\end{aligned}
$$

If $m=7$, then every solution of Equation (3.1) is eventually constant, namely 1, 2, 4, 5, 7, 8, 9 . This holds true because

$$
\begin{array}{lll}
f(1)=1, & f(2)=2, & f(3)=9, \\
f(4)=4, & f(5)=5, & f(6)=9, \\
f(7)=7, & f(8)=8, & \text { and } \quad f(9)=9 .
\end{array}
$$

This completes the proof.

Remark 3.1. Clearly, the modular happy numbers are the pre-images of 1 . For example, if $m=2$, the first modular happy numbers less than or equal to 100 are given by

$\{1,8,10,13,14,15,16,19,22,27,31,38,41,44,45,48,51$,

$54,55,58,61,68,72,77,80,83,84,85,86,89,91,98,100\}$ 


\section{Applications}

The ideas introduced in the proof of Theorem 2.1 can be utilized to investigate the asymptotic behavior of related sequences. For instance, consider the sequence defined recursively as follows.

$$
\begin{aligned}
x_{0}= & d_{k} \cdots d_{1} d_{0}=\sum_{j=0}^{k} d_{j} 10^{j} \\
x_{1}= & g\left(x_{0}\right)=d_{0}+\sum_{j=1}^{k} d_{j}^{j} \\
\vdots \quad & \vdots \\
x_{i+1}= & g\left(x_{i}\right), \quad \text { for } \quad i=0,1,2, \ldots
\end{aligned}
$$

Observe that

$$
\begin{aligned}
& g(n)=n \quad \text { if } \quad k=0 \text {, i.e., } n=d_{0} \\
& g(n)=d_{0}+\sum_{j=1}^{k} d_{j}^{j}=d_{0}+\sum_{j=1}^{k} d_{j} d_{j}^{j-1}<d_{0}+\sum_{j=1}^{k} d_{j} 10^{j}=n \quad \text { if } \quad k=0 .
\end{aligned}
$$

Hence, every solution of Equation (4.1) is eventually constant.

\section{Conclusion}

To identify modular happy number, we investigated the asymptotic behavior of all solutions of Equation (3.1). Furthermore, the same proving tools were applied to Equation (4.1). By doing so, we opened the door for further studies. Indeed, there is myriad of potential investigations. To exemplify, consider the sequence defined recursively as follows.

$$
\begin{aligned}
x_{0}= & d_{k} \cdots d_{1} d_{0}=\sum_{j=0}^{k} d_{j} 10^{j} \\
x_{1}= & h\left(x_{0}\right)=d_{k}+\sum_{j=0}^{k-1} d_{j}^{k-j} \\
\vdots & \vdots \\
x_{i+1}= & h\left(x_{i}\right), \quad \text { for } \quad i=0,1,2, \ldots .
\end{aligned}
$$

\section{Acknowledgment}

We would like to thank Prof. Krassimir T. Atanassov and the reviewers for their suggestions, which improved the exposition of this paper. In particular, we thank Prof. Atanassov for sharing his 2015 paper. 


\section{References}

[1] Atanassov K. (1985) An arithmetic function and some of its applications. Bull. of Number Theory and Related Topics, 9, 18-27.

[2] Atanassov, K. (2015) A digital arithmetical function and some of its applications. Proceedings of the Jangjeon Mathematical Society, 18 (4), 511-528.

[3] Guy, R. (2004) Unsolved problems in number theory (3rd ed.). Springer, New York.

[4] Rosen, K. H. (2012) Discrete Mathematics and Its Applications (7th ed.). McGraw-Hill, New York.

[5] Weisstein, E. W. (2017) Periodic sequence. Available online at http://mathworld. wolfram.com/PeriodicSequence.html. Retrieved March 9, 2017. 